\title{
The role of total wind in the vertical dynamics of ions in the E-region at high latitudes
}

\author{
M. Voiculescu ${ }^{1}$ and M. Ignat ${ }^{2}$ \\ ${ }^{1}$ Department of Physics, University "Dunarea de Jos" of Galati, Romania \\ ${ }^{2}$ Faculty of Physics, University "Al. I Cuza" Iasi, Romania
}

Received: 25 May 2004 - Revised: 20 November 2004 - Accepted: 15 February 2005 - Published: 3 June 2005

\begin{abstract}
A seasonally dependent total neutral wind model obtained from experimental data is used to evaluate the diurnal variation of the vertical ion velocity in the E-region at a high-latitude location (Troms $\varnothing$ ), for each season, in the presence of an electric field with a typical diurnal variation for quiet auroral days. The diurnal variation and spatial locations of the vertical convergence of ions are analyzed and the effect of the total wind on the occurrence of sporadic E-layers is inferred. The results show that the structure of the wind is an important factor in controlling the vertical velocities of ions, favoring or hindering the sporadic E-layer formation. The ion convergence conditions are improved when the permanent wind is removed, which suggests that sporadic E-layers occur when the mean wind has small values, thus allowing the electric field and/or the semidiurnal tide to control the ion dynamics. We conclude that for quiet days the formation of the sporadic layers is initiated by the electric field, while their evolution and dynamics is controlled by the wind. We also find that the seasonal variation of the $E_{s}$ layers cannot be related to the seasonally dependent wind shear. Although we focus on sporadic E-layers, our results can be used in the analysis of other processes involving the vertical dynamics of ions in the E-region at high latitudes.
\end{abstract}

Keywords. Ionosphere (Auroral ionosphere; Ionosphereatmosphere interactions)

\section{Introduction}

Sporadic $\mathrm{E}\left(E_{S}\right)$ layers are thin layers of plasma whose densities are several times higher than the normal density of the Eregion. They form at heights between $100-125 \mathrm{~km}$ and have a lifetime of one to several hours. Two types of layers can be observed at high latitudes: the "auroral" type, which are thick (more than $10-15 \mathrm{~km}$ ) and are produced by precipitating particles, and the so-called "mid-latitude" type $E_{s}$, which have high densities and whose thickness is less than $5 \mathrm{~km}$. In our study we will refer to the last type as $E_{s}$ layers.

Correspondence to: M. Voiculescu

(Mirela.Voiculescu@ugal.ro)
Although the $E_{s}$ layers have been extensively studied over the last five decades, some of their properties and their sporadic character are not totally understood. It has been assumed that the wind shear, which is the main factor of the $E_{S}$ layers formation at middle latitudes (Whitehead, 1989), cannot be very effective at high-latitudes, due to the large inclination of the geomagnetic lines. Nygrén et al. (1984) showed that when the electric field direction lies in the NW sector, ions would ascend at low altitudes and descend at high altitudes, compressing the plasma into dense and thin sheets at $120-125 \mathrm{~km}$. This mechanism is effective at high latitudes, because the values of the electric field are considerably higher. Kirkwood and von Zahn (1991) and Bristow and Watkins (1991) attributed the formation of layers at lower altitudes to the dynamics of the ions under the action of a SW oriented electric field. In this case, ions move downwards with decreasing velocities and accumulate at $90-100 \mathrm{~km}$, where they are at rest, into thicker and less dense $E_{S}$ layers. On the other hand, some studies (Nygrén, 1990; Mathews, 1998; Kirkwood and von Zahn, 1993; Kirkwood, 1997) have shown that the role of the wind in the formation of high altitude $E_{s}$ layers should not be overruled. Along this line, Bristow and Watkins (1993) and Bedey and Watkins (1996) have considered the $E_{s}$ generation in some particular cases using models for the wind. A comprehensive study of highlatitude $E_{S}$ (Kirkwood and Nilsson, 2000) presents numerical simulations of $E_{s}$ layers resulting from the action of the wind and the electric field, both separately and together, but considering only the semidiurnal tide. Nevertheless, the real atmosphere involves a mean invariable wind and a superposition of irregular tidal winds, whose values, amplitudes or phases are not always consistent with the models (Brekke et al., 1994). Despite the extensive studies of $E_{s}$ generation at high latitudes, there is no sufficient evidence to clarify the contribution of each factor to the formation of $E_{s}$ and what surrounding conditions are needed for one or the other mechanism to prevail.

The aim of this paper is to investigate the total wind contribution to $E_{s}$ layer formation for quiet days in the presence of a medium electric field, in order to obtain a more realistic image of the wind's role in the $E_{s}$ layers' occurrence. To 
our knowledge, there are no studies referring to the relation of high latitude $E_{s}$ with the total wind, which includes the tides and the background wind, in conjunction with the electric field. Existing papers, either theoretical or experimental, generally neglect the prevailing wind because the wind-shear mechanism is based on the tidal variability of the neutral motion with the altitude. We will calculate the diurnal variation of vertical ion velocity in the presence of a total wind and estimate the effect of the background wind on the vertical motion of ions and, consequently, on the $E_{s}$ formation. The wind values, comprising the mean wind and all the tides, are derived from data obtained by Brekke et al. (1994) from EISCAT measurements for $70^{\circ} \mathrm{N}, 19^{\circ} \mathrm{E}$.

There are also other uncertainties that make the $E_{s}$ layers a controversial subject. The physical reasons for the $E_{s}$ layers' diurnal and especially seasonal distributions are still under debate. Dense $E_{S}$ layers are observed much more often during late afternoon (local time) and, to a lesser extent, in the morning, than in the rest of the day (Turunen, 1976; Kirkwood, 1997; Kirkwood and Nilsson, 2000). Polar cap $E_{S}$ seem to have one night peak (Bedey and Watkins, 1998). The annual variation of $E_{s}$ occurrence has a distinct peak during summer, a lower one (less than $10 \%$ of the summer occurrence) during winter and a minimum at equinoxes ( $\mathrm{Tu}-$ runen, 1976; Bedey and Watkins, 1996; Kirkwood, 1997; Kirkwood and Nilsson, 2000; MacDougall et al., 2000). The results of Brekke et al. (1994) show that the total wind varies with the season, so that the results presented in this paper give us the opportunity to verify whether the seasonal distribution of $E_{s}$ layers could be related to the seasonal variation of the wind. Since there are no reports of seasonal variations of the mean electric field, except for an increase during winter (Kirkwood, 1997), the electric field diurnal variation is assumed to be the same for each season. Since a large electric field (more than $30 \mathrm{mV} / \mathrm{m}$ ) would most likely dominate the wind effects on ions at high latitudes (Kirkwood, 1997; Kirkwood and von Zahn, 1991; Bristow and Watkins, 1991), we will focus in the present paper on the wind contribution and consider only the situations when the electric field has a typical structure (during a quiet day) and average values. In this paper we calculate only the vertical velocity of ions without going further to simulate the $E_{s}$ layer formation. Our discussion refers to $E_{S}$ formation on the basis that poor/strong ion convergence means weak/dense number of layers, provided that the metallic ion content remains unchanged.

\section{Method}

The ion velocity formula for one species of ions, where the gravitational term is ignored, is (e.g. Kelley, 1989):

$$
\begin{aligned}
\boldsymbol{V}_{\boldsymbol{i}}= & \frac{1}{1+\beta_{i}^{2}}\left\{\boldsymbol{U}+\beta_{i}(\boldsymbol{U} \times \boldsymbol{b})+\beta_{i}^{2} \boldsymbol{b} \cdot(\boldsymbol{U} \cdot \boldsymbol{b})-\right. \\
& -\frac{k_{B} T_{i}}{m_{i} \Omega_{i}} \beta_{i}\left[\frac{\nabla N}{N}+\beta_{i}\left(\frac{\nabla N}{N} \times \boldsymbol{b}\right)+\beta_{i}^{2} \boldsymbol{b} \cdot\left(\frac{\nabla N}{N} \cdot \boldsymbol{b}\right)\right] \\
& +\frac{e m_{i} \Omega_{i}}{\beta}{ }_{i}\left[\boldsymbol{E}+\beta_{i}(\boldsymbol{E} \times \boldsymbol{b})+\beta_{i}^{2} \boldsymbol{b} \cdot(\boldsymbol{E} \cdot \boldsymbol{b})\right],
\end{aligned}
$$

where $\boldsymbol{b}=\boldsymbol{B} / \mathrm{B}$ is the geomagnetic unit vector, $\boldsymbol{U}$ is the neutral wind, $\boldsymbol{E}$ is the total electric field (polarization plus external field), $N$ is the plasma density, $\Omega_{i}, v_{i n}, m_{i}$ and $T_{i}$ are the ion gyrofrequency, the ion-neutral collision frequency, the ion mass and the ion temperature, respectively, and $\beta_{i}=\Omega_{i} / \nu_{i n}$. Sporadic E-layers are very narrow in the vertical direction and have large horizontal dimensions, so that only the vertical component of the ion velocity will be considered, although Nygrén et al. (1990) pointed out that horizontal transport might sometimes have a decisive impact over the formation time.

In a geographical coordinate system with the vertical $\mathrm{z}$ axis positive upward and the $\mathrm{x}$-axis to the east, the ion velocity in the vertical direction, neglecting molecular and ambipolar diffusion, will be given by:

$$
\begin{aligned}
V_{i z}= & \frac{1}{1+\beta_{i}^{2}}\left[\left(1+\beta_{i}^{2} \sin ^{2} I\right)\left(U_{z}+\frac{e}{m_{i} v_{i}} E_{z}\right)\right. \\
& +\beta_{i}\left(U_{E}+\frac{e}{m_{i} v_{i}} E_{E}\right) \cos I- \\
& \left.-\beta_{i}^{2}\left(U_{N}+\frac{e}{m_{i} v_{i}} E_{N}\right) \sin I \cos I\right],
\end{aligned}
$$

where $I$ is the inclination of the geomagnetic field line, $U_{E}$, $U_{N}, U_{z}$ and $E_{E}, E_{N}$ and $E_{z}$ are the horizontal eastward, northward and vertical components of the wind, respectively, of the external electric fields. The diffusion and the polarization electric fields have been neglected here because for steady-state conditions, these two terms contribute more to the width, peak density and lifetime of the layer and less to its formation. The diurnal evolution of the vertical dynamics of ions will be illustrated by calculating their velocities at altitudes separated by $0.5 \mathrm{~km}$, at a time step of $15 \mathrm{~min}$.

The three geographical components of the total wind as a function of time (in hours) can be expressed as:

$$
\begin{aligned}
U_{E, N, z}(t) & =U_{0 E, N, z}+U_{24 E, N, z} \cos \left(\frac{2 \pi}{24} t+\phi_{24 E, N, z}\right) \\
& +U_{12 E, N, z} \cos \left(\frac{2 \pi}{12} t+\phi_{12 E, N, z}\right) \\
& +U_{8 E, N, z} \cos \left(\frac{2 \pi}{8} t+\phi_{8 E, N, z}\right) \\
& +U_{6 E, N, z} \cos \left(\frac{2 \pi}{6} t+\phi_{6 E, N, z}\right)
\end{aligned}
$$

where $U_{0 E, N, z}$ are the mean velocities of the zonal, meridional and vertical mean winds, and $U_{T E, N, z}$, and $\phi_{T}$, with $T=24,12,8$ and 6, are the amplitudes and, respectively, the phases of the zonal, meridional and vertical components of the tides.

We derived the values of the wind in the 95-125 km range by means of a cubic spline interpolation of the experimental values obtained by Brekke et al. (1994) for a number (4, 5 or 6 , depending on the season) of fixed altitudes during quiet auroral ionosphere $\left(A_{p}<16\right)$. Using the ion velocity vectors measured by EISCAT at $69.5^{\circ} \mathrm{N}$ latitude through 7 days in spring, summer and autumn and 3 days in winter, they calculated the background wind and the amplitudes and phases of tides with periods of $24,12,8$ and $6 \mathrm{~h}$. The cubic spline is a curve defined by a set of three order polynomials, 

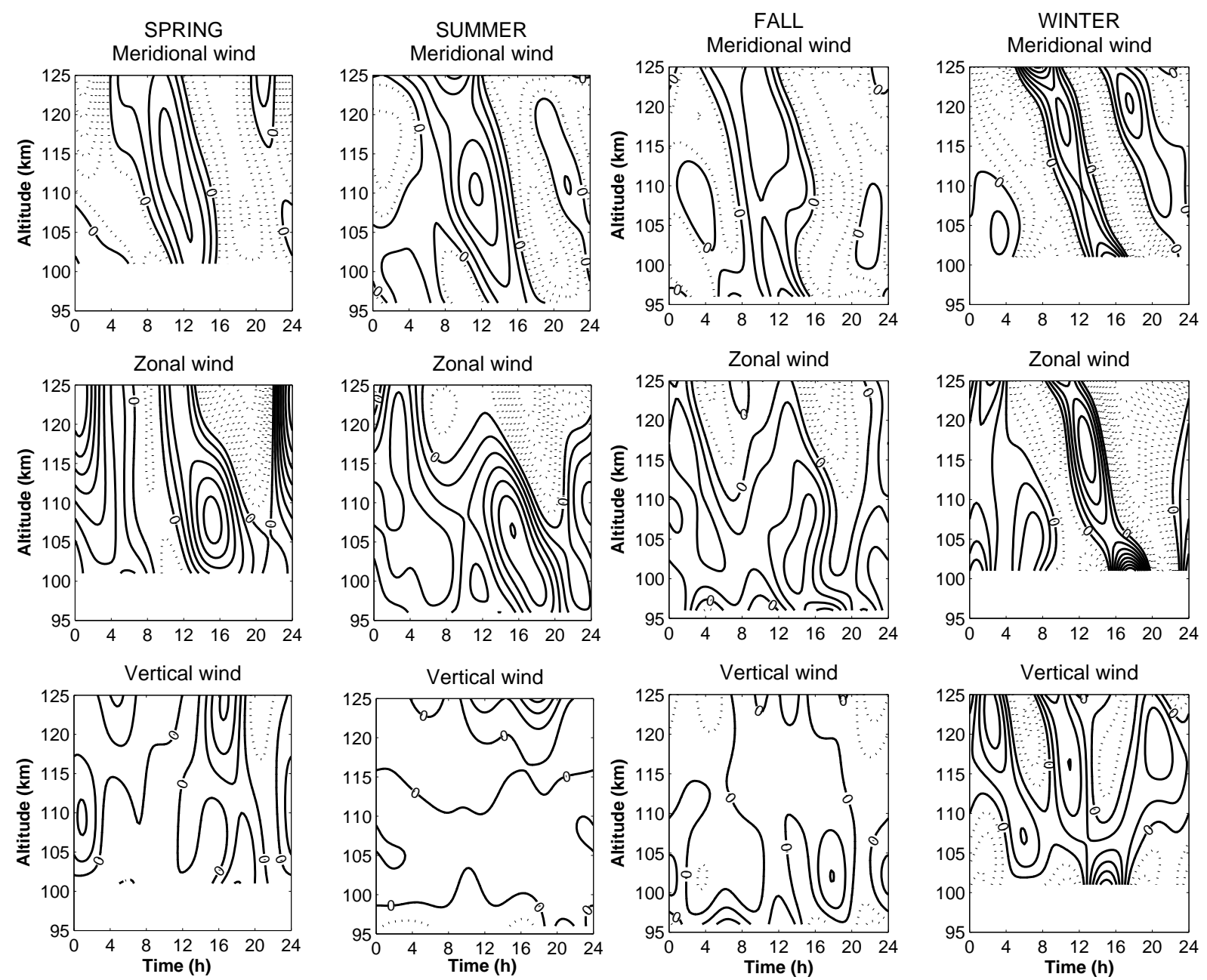

Fig. 1. Diurnal variation of the wind components for spring, summer, fall and winter. The spacing interval between lines is $20 \mathrm{~m} / \mathrm{s}$ for the horizontal components and $5 \mathrm{~m} / \mathrm{s}$ for the vertical component. Continuous lines for positive (eastward, northward, up) values, dotted lines for negative values (in spring and winter the lowest height where data were available was $101 \mathrm{~km}$ ).

where every adjoining pair of points is connected by a different 3-order polynomial. It can be applied when average values are demanded and when the data points are well separated, as they are in our case. The driving criteria are that the curve is smooth and that it passes through every point (Borse, 1997), which holds in our study, since winds cannot change too abruptly with increasing altitude. The results for the meridional, zonal and vertical wind are displayed in the form of contour plots in Fig. 1 for spring, summer, fall and winter. These are the wind values that will be used to estimate the evolution of the vertical ion velocity in time.

The meridional, zonal and vertical components of the electric field were obtained by averaging the data from Brekke et al. (1994) and Kirkwood (1997), which are representative for the variation of the electric field during quiet days. We have used electric field data from these two sources in order to gain a more general picture of the effect of the wind on the ion convergence. Their time dependence over $24 \mathrm{~h}$ is plotted in Fig. 2.
The collision frequency is (Banks and Kockarts, 1973):

$$
\begin{aligned}
v_{\text {in }}= & {\left[\frac{1.80}{\sqrt{A(A+28)}} \cdot n_{N_{2}}+\frac{1.83}{\sqrt{A(A+32)}} \cdot n_{O_{2}}\right.} \\
& \left.+\frac{0.91}{\sqrt{A(A+16)}} \cdot n_{0}\right] \cdot 10^{-14},
\end{aligned}
$$

where the neutral densities of $\mathrm{N}_{2}, \mathrm{O}_{2}$ and $\mathrm{O}$ are taken from the MSIS-90 (Hedin, 1991) model run for January, April, July, October, day $15,12: 00 \mathrm{UT}$, for $70^{\circ} \mathrm{N}$ and where the ion atomic mass is considered $\mathrm{A}=56(\mathrm{Fe})$, keeping in mind that the sporadic E-layers' ionic composition is mainly metallic. The difference in neutral densities between night and day at these altitudes causes a very small variation of the ion-neutral collision frequency. Note that the inclusion of a small percentage of molecular ions would not essentially modify the collision frequency. 


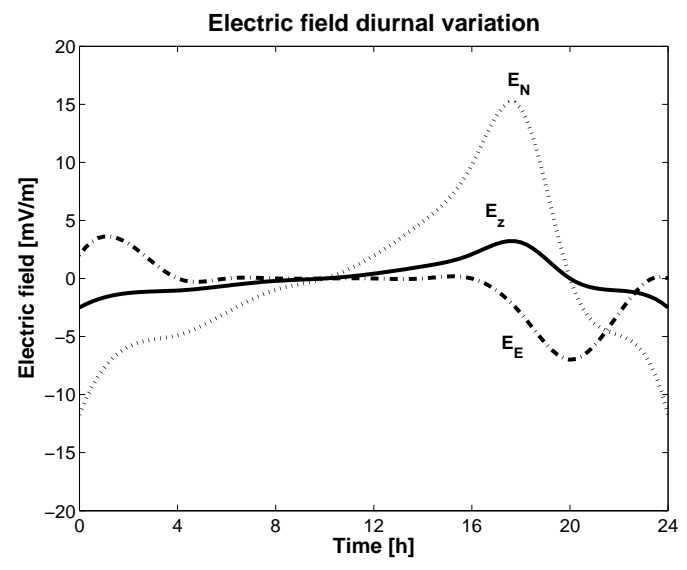

Fig. 2. Electric field components diurnal variations: $E_{N}$ (meridional): dotted, $E_{E}$ (zonal): dash-dotted, $E_{z}$ (vertical): continuous line.

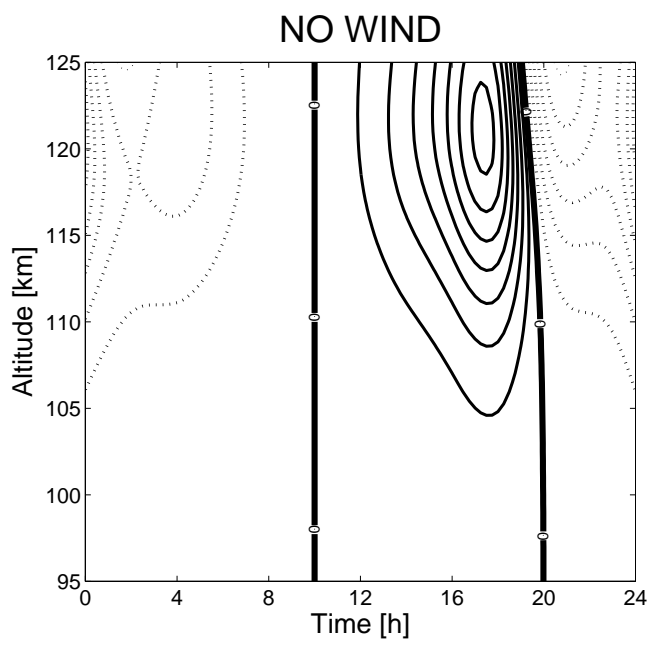

Fig. 3. Vertical velocity field for ions in the presence of the electric field; spacing interval $5 \mathrm{~m} / \mathrm{s}$. Continuous/dotted lines represent positive/negative velocities, corresponding to upward/downward movement. Zero lines are thickened.

\section{Results and discussions}

We start by neglecting the wind and show in Fig. 3 the diurnal variation of the vertical velocity of ions under the action of the electric field. Sporadic E-layers could form between 19.00-20.00 LT, but the convergent zero line descends very fast. By convergent zero line we mean the line connecting those points, $z_{c}$, where the ions have zero velocities and $\left(d v_{i z} / d z\right)_{z c}$ is negative. If formed, the layer would descend with a velocity of $20-25 \mathrm{~km} / \mathrm{h}$, a value that has no counterpart in the experimental data. Around local midnight, the downward motion of ions could result in layers at $105 \mathrm{~km}$, but these would probably be thick, since ions are at rest over a range of more than $10 \mathrm{~km}$. Thus, an electric field having a typical quiet diurnal variation could form a layer, but its dynamics would not be consistent with the observations.
The vertical dynamics of ions under the action of the wind when the electric field is neglected can be seen in Fig. 4. The vertical convergence seems to be effective in all seasons except for spring, when the "convergent" zero line descends almost as fast as in the previous case. The structure of the wind favors the accumulation of ions in the morning and late afternoon in summer and fall, but the velocities are rather small. The layers, if formed, would be thick and with low peak densities. On the other hand, their dynamics would be closer to what we know about $E_{s}$ vertical movement, i.e. they reside for a while at the altitude where they have formed or descend slowly (Turunen, 1976; Kirkwood; 1997; Kirkwood and Nilsson, 2000). When only the wind is present, thin layers could form during winter mornings and afternoons around $110-115 \mathrm{~km}$, when our results show that the vertical velocities are large enough. An interesting aspect is the upward slope of the convergence line between $14 \mathrm{~h}$ and $18 \mathrm{~h}$ in wintertime, which could account for the observations of some ascending sporadic E-layers (Kirkwood, 1997, Turunen et al., 1993). An overall conclusion is that the structure of the wind favors the vertical accumulation of ions (horizontal or slow descending convergence lines) but, except for winter, their vertical movements are very slow. This means that dense and thin layers could not form under the action of the total wind.

Figure 5 summarizes the results when both electric field and wind are included. A first common characteristic is the afternoon-evening zero line starting around $120 \mathrm{~km}$ at $16 \mathrm{~h}$, providing conditions for effective ion accumulation in each season. The altitudes where metallic ions could converge vary between 110 and $125 \mathrm{~km}$, higher for afternoon and lower for later hours. Due to the inclusion of the electric field, whose values are large between 16 and 24 LT, the velocities of ions increase appreciably. The vertical profiles found in Fig. 4 are, generally, maintained.

The spring plot shows no other favorable time for $E_{s}$ occurrence, while in fall the layers could form around $105 \mathrm{~km}$ during nights. In summer there is a zero line around $115 \mathrm{~km}$, residing from the early morning hours until midday, but the downward velocities above this line are small and beneath this altitude the ions seem to be at rest, meaning that $E_{S}$ layers that could form would be thick and weak. However, an increase of the downward ion velocity in this time interval enhances the probability of layer formation. Propitious conditions for the ion convergence in the pre-noon sector at altitudes of $110-115 \mathrm{~km}$ are observed, again in winter. This agrees with the experimental observations showing that many of the winter $E_{S}$ layers form in the mornings at higher altitudes, after which they descend and merge into a unique layer around noon (Kirkwood, 1997; Kirkwood and Nilsson, 2000). While the formation and descent of the initial layers can be interpreted as a result of the descending zero line between $6 \mathrm{~h}$ and $10 \mathrm{~h}$, the broad winter noon layers found by Kirkwood (1991) have no counterpart in the dynamics of the ions. According to our results, low altitude layers, which form a large part of the observed $E_{s}$ events, have no dynamical conditions to form. 

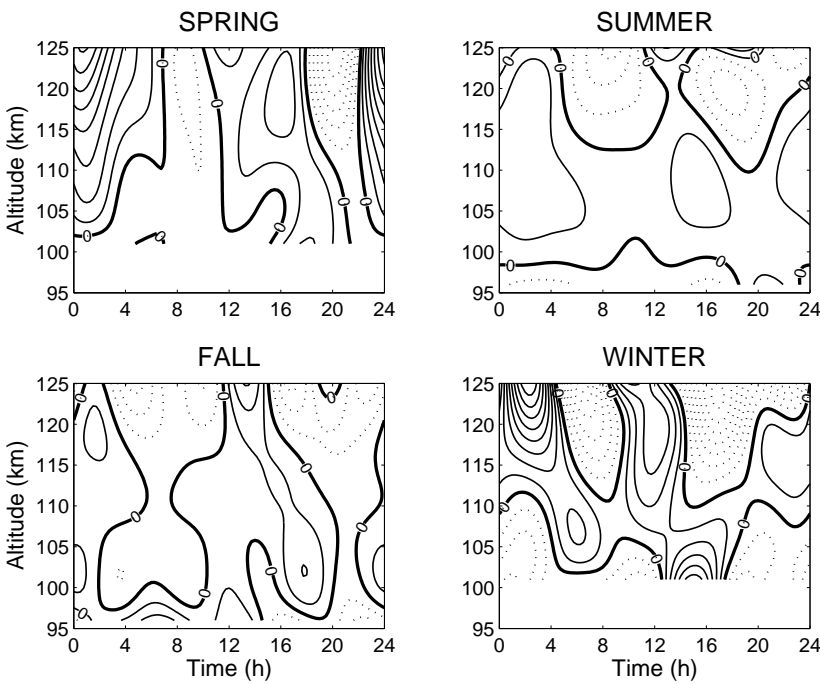

Fig. 4. Vertical velocity field resulting from the action of the wind for spring, summer, fall and winter; the same plot details as in the previous figures.

A comparison between Figs. 3 and 4, and, respectively, 5 , reveals that in the lower E-region, the profiles are shaped by the wind during the entire day. The contribution of the electric field in this region is seen in the slight modification of the velocities. In the upper part this observation still holds, but the role of the electric field is definitely more important not only in the increase of the absolute values of velocities, but also in shaping the altitude-time profiles.

Figures 1 and 2 show that both the wind and the electric field have a structure that favors the vertical convergence of ions in each season's afternoon. The zonal wind structure shows an EW (east below, west above) wind shear, which could form sporadic E-layers (Whitehead, 1989). The electric field has a NW orientation favorable to the high-latitude $E_{s}$ formation (Nygrén et al., 1984). The examination of the velocity profiles depicted in Fig. 5 shows that, if formed, the layer would descend with about $2 \mathrm{~km} / \mathrm{h}$. Note that this value is close to the downward velocity of a layer produced by a 12-h tide found by Kirkwood and Nilsson (2000) in their numerical simulations. However, the wind alone would not be effective in the formation of the layer, as one can see in Fig. 4. These observations suggest that both factors have to be considered when analyzing the evolution of the $E_{s}$ layer, each of them contributing differently to the formation and evolution of the $E_{s}$ layer. The electric field seems to be responsible for the effective convergence of the ions, thus for the initialization of the layer formation at high altitudes, whereas the wind controls its dynamics after the formation. The importance of the wind is endorsed by the fact that although the electric field diurnal variation is the same, the conditions for ion accumulations differ from season to season.

Studies of $E_{s}$ occurrence in connection with electric fields found that layers were absent during favorable electric field conditions (e.g. Nygrén, 1989; Bristow and Watkins, 1993;
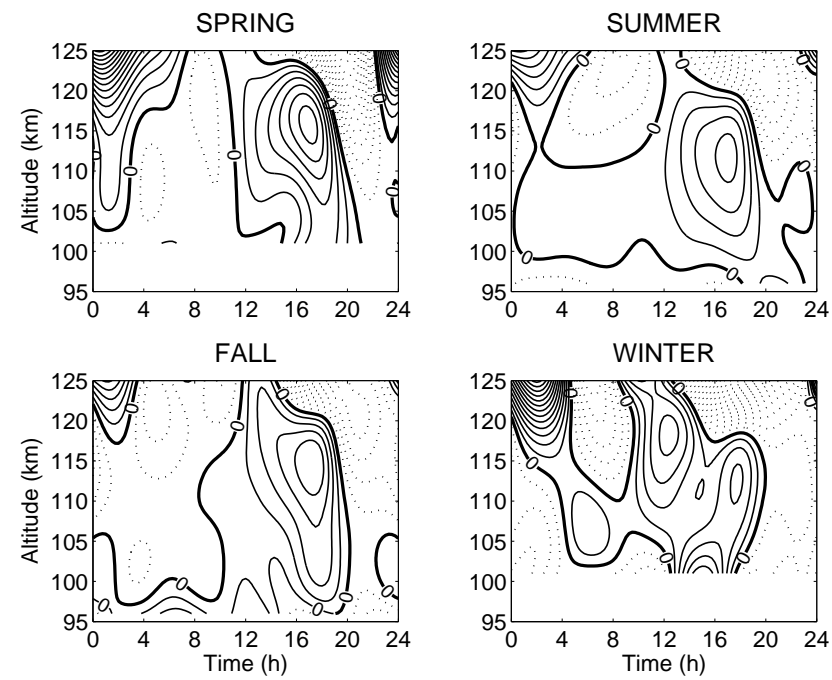

Fig. 5. Vertical velocity field in the presence of both wind and electric field for spring, summer, fall and winter; the same plot details as in the previous figures.
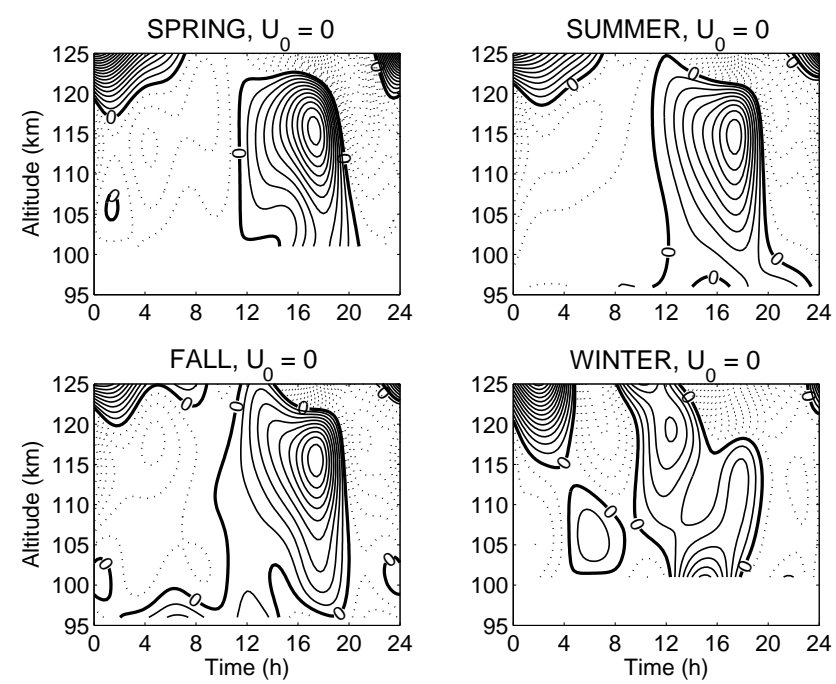

Fig. 6. Vertical velocity field when the background wind is removed for each of the four seasons; the same plot details as in the previous figures.

Mathews, 1998; Kirkwood and Nilsson, 2000). Our results show that, for the given structure of the wind, the dynamics of ions could not result in the formation of layers at low altitudes, where a large part of the $E_{S}$ layers' are observed. This conclusion supports the suggestion that one of the factors responsible for the $E_{s}$ layers absence during the appropriate direction of the electric field might be an opposing wind ( $\mathrm{Ny}$ gren, 1990; Mathews, 1998; Kirkwood and Nilsson, 2000). Since a semidiurnal tide has been found to be effective in the formation of $E_{s}$ layers, we removed the background wind and plotted the diurnal variation of the resulting vertical velocity of ions in Fig. 6. The noticeable increase of velocities seen in this case is equivalent to an increased probability of 
$E_{s}$ layer formation. Moreover, the convergence conditions are also improved at low altitude levels. We conclude that $E_{s}$ occurrence at low altitudes is possible only if the background wind is noticeably diminished, allowing the electric field or the 12 -h tide to control the ions at low altitudes. The comparison between the vertical velocities of the ions in the presence of the mean wind with those obtained after excluding it suggests that the background wind impedes the formation of $E_{s}$ layers.

Certainly, our results have limitations and are affected with errors. Keeping in mind that we consider only days when the electric field has mean values and no fluctuations, a rough estimation shows that the velocities would vary with $3-5 \mathrm{~m} / \mathrm{s}$ at higher altitudes and $10-15 \mathrm{~m} / \mathrm{s}$ at low altitudes. Accordingly, the change in the convergence heights would be of $1-2 \mathrm{~km}$ close to those points where the velocity gradient is the highest, specifically, in the upper part of the E-region during the second part of the day. At low altitudes and/or during midday the variation would be larger, reaching $7-10 \mathrm{~km}$ in those regions where the ions move very slow. In the absence of the background wind, the velocities and, consequently, the convergence heights, are less affected, due to the increase in the vertical gradient of the velocity both at high and low altitudes.

For all four seasons, the vertical convergence of ions in the afternoon-evening interval can be correlated with the evening maximum in the diurnal distribution of $E_{s}$ layers found in ionosonde (Turunen, 1976) and ISR (Kirkwood, 1997) observations. Moreover, the shape of the descending convergence lines, which start and reside for some time around $120 \mathrm{~km}$, confirms the results of the same authors, which indicate that the mean heights of afternoon Es layers are the highest of all day.

Some features of the $E_{s}$ morphology can be recognized in the seasonal variation of the ion convergence conditions, but they are few and unconvincing. The lack of conditions during spring and the increased possibility of $E_{s}$ formation during summer and winter correspond to the absence of $E_{S}$ layers during spring and to the maximization of $E_{s}$ occurrence during summer. In opposition, the fall minimum and the lower maximum in winter of the $E_{s}$ activity cannot be explained by the seasonal variation of the convergence conditions. More, evening layers in fall are equally probable as in summer at the same height. Although the diurnal variation of the vertical ion velocity changes from spring to winter, the differences between seasons are small and, besides, not in phase with the observed seasonal characteristics of the $E_{s}$ occurrence. Our calculations support the hypothesis that the summer maximum of $E_{s}$ activity could be connected to the seasonal variation of the metallic ion content (Bedey and Watkins, 1997) or to the seasonal variation of the neutral components and/or chemical rates, which, in turn, would modify the collision frequency (Mathews, 1998; Kirkwood and Nilsson, 2000). Experimental results and theoretical investigations are needed, however, to confirm which of these theories could account for the $E_{s}$ morphology.

\section{Conclusions}

When both electric field and total wind are considered for a location at $70^{\circ} \mathrm{N}, 19^{\circ} \mathrm{E}$, sporadic E-layers could occur during afternoon and evening hours at high altitudes, regardless of the season. The dynamical conditions required for the formation of $E_{s}$ layers are not fulfilled at low altitudes, where the majority of layers have been experimentally observed. According to our results, the sporadic layers are produced by an electric field with the appropriate orientation, while their dynamics is controlled by the wind structure. The most intriguing result is that in summer, when the maximum of $E_{S}$ activity is recorded, the ion accumulation is not very efficient, especially below $115 \mathrm{~km}$. When the background wind is removed, the dynamical conditions for the formation of $E_{s}$ layers are improved, especially in the lower E-region. The velocity gradients increase at all altitudes, meaning that in this case $E_{s}$ could form easier. We conclude that the probability of dense layers to occur is greatly reduced when the background wind is significant.

The experimentally observed diurnal variation of the $E_{S}$ layers occurrence is in phase with the ion vertical convergence under the combined action of the wind and the electric field. The evening maximum of $E_{s}$ could be explained by the existence of vertical convergence of ions during the same interval. On the other hand, our calculations show that the seasonal variation of the wind shear cannot be responsible for the $E_{S}$ morphology. Some of the characteristics of the $E_{s}$ seasonal variation can be identified in our results, while others do not agree with the experimental findings. Since ion accumulation conditions do not have the expected seasonal variation and given that no other force is yet known to contribute to the sporadic layer formation, we support the suggestion that the seasonal variation of $E_{S}$ might originate in a related variation of the metallic ion content, collision frequency and/or chemical reactions.

We are aware of the fact that our results refer to averaged conditions and that the wind calculations are affected by errors generated by the relatively small number of measurements and by interpolation. Furthermore, high latitude days are not regularly quiet. However, the different profiles of the ion velocity obtained for the four seasons, for the same electric field, and the modifications in the ion velocities induced by changes in the structure of the wind confirm that winds, particularly their invariable component, should be considered as an important factor in the occurrence of $E_{S}$ layers at high latitudes.

The diurnal variation of the vertical velocity of the ions which were obtained were used in the present paper to infer the contribution of the wind relative to the electric field, to the formation and evolution of sporadic E-layers. We emphasize that our results could be analyzed with respect to any other process that takes place in the high-latitude E-region and involves the vertical dynamics of ions. 
Acknowledgements. We thank T. Nygrén and T. Bösinger for helpful comments on the paper. This work has been initiated during a visit of one author (M. Voiculescu) to the University of Oulu, supported through a Marie Curie research training fellowship by the European Community.

Topical Editor M. Lester thanks two referees for their help in evaluating this paper.

\section{References}

Banks, P. M. and Kockarts, G.: Aeronomy. Part A, Academic Press inc., New York, NY, 1973.

Bedey, D. F. and Watkins, B. J.: Seasonal occurrence of thin metallic ion layers at high latitudes, Geophys. Res. L., 23, 2789-2792, 1996.

Bedey, D. F. and Watkins, B. J.: Large-scale transport of metallic ions in the lower ionosphere, J. Geophys. Res. S. P., 102(A5), 9675-9681, 1997.

Bedey, D. F. and Watkins, B. J.: Diurnal occurrence of thin metallic ions layers in the high latitude ionosphere, Geophys. Res. L., 25(20), 3767-3770, 1998.

Borse, G. J.: Numerical methods with MATLAB: A resource for Scientists and Engineers, PWS Publ. Comp., 1997.

Brekke, A., Satonori, N., and Sparr, T: .Studies of the E-region neutral wind in the quiet auroral ionosphere, J. Geophys. Res. S. P., 99, 8801-8825, 1994.

Bristow, W. A. and Watkins, B. J.: Numerical simulation of the formation of thin ionization layers at high latitudes, Geophys. Res. L., 18, 404-407, 1991.

Bristow, W. A. and Watkins, B. J.: Incoherent scatter observation of thin ionisation layers at Sondreström, J. Atmos. T. P., 55, 873894, 1993.

Hedin, A. E.: Extension of the MSIS thermosphere model into the middle and lower atmosphere. J. Geophys. Res. S. P., 96, 11591172, 1991.

Kelley, M. C.: The Earth Ionosphere: Plasma Physics and Electrodynamics, Academic Press, San Diego, California, 1989.

Kirkwood, S.: Anomalous ion layers in the high-latitude winter Eregion, Geophys. Res. L., 18, 1189-1192, 1991.
Kirkwood, S: Thin ion layers in the high-latitude lower ionosphere, ADV Space R., 19 (1), 149-158, 1997.

Kirkwood, S. and von Zahn, U.: The role of magnetospheric electric fields in producing auroral zone thin layers, ADV Space R., 12(10), 225-228, 1991.

Kirkwood, S. and von Zahn, U.: Formation mechanisms for lowaltitude $E_{S}$ and their relationship with neutral Fe layers: Rresults form the METAL campaign, J. Geophys. Res. S. P., 98, 21 549$21561,1993$.

Kirkwood, S. and Nilsson, H.: High-latitude sporadic-E and other thin layers - the role of magnetospheric electric fields, Space Sci. R., 91, 579-613, 2000.

MacDougall, J. W., Plane, J. M. C., and Jayachandran, P. T.: Polar cap sporadic E: Part 1, Observations J. Atmos. S.-P., 62, 11691176, 2000.

Mathews, J. D.: Sporadic E: Current views and recent progress, J Atmos S.-P., 60, 413-435, 1998.

Nygrén, T., Jalonen, L., Oksman, J., and Turunen, T.: The role of electric field and neutral wind direction in the formation of sporadic E-layers, J. Atmos. T. P., 46, 373-381, 1984.

Nygrén, T.: Studies of sporadic E-layer using the EISCAT incoherent scatter radar, Adv. Space R., 9(5), 73-81, 1989.

Nygrén, T.: Vertical plasma transport due to electric fields and neutral winds in the auroral ionosphere, Adv. Space R., 10(6), 297305, 1990.

Nygrén, T., Lanchester, B. S., Huuskonen, A., Jalonen, L., Turunen, T., Rishbeth, H., and van Eyken, A. P.: Interference of tidal and gravity waves in the ionosphere and an associated sporadic Elayer, J. Atmos. T. P., 52, 609-623, 1990.

Turunen, T: The diurnal variation of $E_{S}$ layers parameters at Sodankylä in summer 1973 based on ionospheric soundings utilizing low fixed gain, Geophysica, 14, 77-98, 1976.

Turunen, T., Nygrén, T., and Huuskonen, A.: Nocturnal highlatitude E-region in winter during extremely quiet conditions. J. Atmos. T. P., 55, 783-795, 1993.

Whitehead, J. D.: Recent work on mid-latitude and equatorial sporadic-E, J. Atmos. T. P., 51, 401-424, 1989. 